\title{
RETO AL INGENIO PROBABILISTICO: UNA PROPUESTA DIDACTICA PARA LA ENSEÑANZA-APRENDIZAJE DE LAS PROBABILIDADES
}

\section{PROBABILISTIC CHALLENGE TO WIT: A PROPOSAL FOR TEACHING BY THE TEACHING - LEARNING PROBABILITIES}

\author{
Elizabeth Gandica-de Roa ${ }^{1}$
}

Forma de citar: GANDICA-DE ROA Elizabeth. Reto al ingenio probabilistico: una propuesta didactica para la eneseñanza-aprendizaje de las probabilidades. Eco.Mat. 2014; 5(1): 66-73.

Recibido:

Agosto 28 de 2014

Aceptado:

Octubre 30 de 2014

\section{RESUMEN}

Esta investigación se orienta hacia la utilización de juegos inéditos de mesa, como herramienta metodológica de apoyo al proceso de enseñanza de probabilidades, propuestos por estudiantes de Estadística en Ingeniería Industrial de la Universidad Experimental Nacional del Táchira (UNET).

El proyecto surge como estrategia para mejorar las dificultades de aprendizaje de modelos de probabilidad, dirigido a disminuir el alto índice de reprobados en el tema, además de ampliar las potencialidades intelectuales y creativas del alumno, involucrándole en un proceso recreativo que impulsa habilidades de razonamiento, creatividad y procesamiento lógico estadístico. Diversos autores han mostrado que a través del diseño de juegos de mesa didácticos, enfocados a "aprender jugando", se puede lograr un aprendizaje significativo, divertido y prolífero.

Además de inducir creatividad e ingenio, los cuales constituyen pilares fundamentales en los estudios de Ingeniería. En este ambiente, los estudiantes inventan el juego de azar, establecen sus reglas, diseñan sus entidades y tablero, y plantean interrogantes mediante la identificación de relaciones entre el juego y los conceptos de teoría de probabilidades aprendidos durante las clases impartidas. El juego debe contener distintos planteamientos teóricos, los cuales requieren ser resueltos por los jugadores para avanzar en el mismo.

A través del desarrollo del juego se realizan evaluaciones sobre conceptos propios de la asignatura, lo cual resulta en una propuesta didáctica innovadora, que estimula el aprendizaje y adquisición de conocimiento que ayuda a reducir temores hacia el cálculo e interpretación de los eventos probabilísticos.

Palabras Clave: Actividad Lúdica, Teoría de Probabilidades, Estrategias de enseñanza 


\section{ABSTRACT}

Enero-Diciembre 2014

This research is oriented towards the use of unpublished games, as a methodological tool to support the teaching of probabilities, given by students of Statistics in Industrial Engineering from the National Experimental University of Táchira (UNET).

The project emerged as a strategy to improve the learning difficulties of probability models, aimed at reducing the high rate of reprobates on the topic, and expand the intellectual and creative potential of students, involving them in a recreational process that promotes reasoning skills, statistical creativity and logic processing. Several authors have shown that through the design of educational games, focused on "learning through play", you can achieve a significant, prolific and fun learning. In addition to inducing creativity and ingenuity, which are fundamental pillars in engineering studies.

In this environment, students invent gambling, establish its rules, its entities and board design, and raise questions by identifying relationships between the game and the concepts of probability theory learned during lectures given. The game should contain different theoretical approaches, which need to be resolved by the players to advance on it. Through game evolution, concepts from the course are studied, resulting in an innovative methodological approach which encourages learning and acquiring knowledge that helps to reduce fears to calculation and interpretation of probabilistic events.

Keywords: Playful Activity, Probability Theory, Teaching Strategies

\section{INTRODUCCIÓN}

U na de las funciones del juego es consolidar las estructuras intelectuales a lo largo del proceso en el que se van adquiriendo, Piaget (1986). Jugar bajo experimentación lúdica controlada, es una manera entretenida de aprendizaje útil, orientada a entender y comprender el funcionamiento de sucesos aleatorios de fácil construcción, mediante elementos populares conocidos.

El juego genera aprendizajes significativos como lo plantea David Ausbel (2009), quien fue el precursor de esta teoría que ha servido para apuntalar la enseñanza en todas las materias.

Los resultados arrojados por las investigaciones realizadas con anterioridad por psicólogos, sociólogos, pedagogos, entre otros, demuestran que el juego es un valioso medio para educar al ser humano y fomentar su desarrollo integral: físico, moral, intelectual, actitudinal, de lenguaje y psicomotriz. Es un recurso que favorece y despierta el interés del participante en la adquisición de conocimientos. 
No. 1

Enero-Diciembre 2014 ISSN 1794-8231
Con la lúdica se enriquece el aprendizaje por el espacio dinámico y virtual que implica, como espejo simbólico que transforma lo grande en pequeño, lo chico en grande, lo feo en bonito, lo imaginario en real y a los alumnos en profesionistas. El elemento principal, del aprendizaje lúdico, es el juego, recurso educativo que se ha aprovechado muy bien en los niveles de la educación básica y primario $\mathrm{y}$ enriquece el proceso de enseñanza-aprendizaje. Puede emplearse con una variedad de propósitos, dentro del contexto de aprendizaje, pues construye autoconfianza e incrementa la motivación en el alumno. Es un método eficaz que propicia lo significativo de aquello que se aprende. Martínez (2008)

En una instrucción para y por la recreación, el juego en la modalidad de educación especial contribuye a despertar en los jóvenes la verdadera conciencia individual y colectiva, y este convencimiento puede ser considerado por los docentes, para desempeñar un rol decisivo en el proceso de enseñanza-aprendizaje; que implemente esta actividad como herramienta didáctica pedagógica, en los diferentes espacios de instrucción que conforman el aula.

En el nivel de Educación Superior, los juegos no han sido incluidos como elementos formadores, aun cuando es innegable que pueden constituir un poderoso instrumento de aprendizaje con valor agregado cultural importante, constituyendo una herramienta que despierta la construcción del sentido y conceptos probabilísticos, además de ayudar a superar barreras psicológicas o temores hacia el cálculo de probabilidades, y estimular habilidades de observación, razonamiento, deducción, análisis, experimentación e interpretación de conceptos teóricos.

La actividad lúdica que conlleve connotaciones de tipo tradicional, debe estar a cargo del docente, pues se considera que es la persona que puede aprovechar las situaciones que surjan del contacto cotidiano con los jóvenes; para involucrarlos en el ejercicio de actividades, que además de fortalecer su desarrollo integral, les inculque interés por el conocimiento y apasionamiento por la búsqueda de información, con la finalidad de fortalecer su capacidad de comprensión numérica.

El juego didáctico ofrece al alumno un trabajo intelectual activo, que le lleva a evaluar distintas posibilidades de estrategias y jugadas. Proporciona al estudiante planeamiento, raciocinio lógico, operaciones de cálculo probabilístico, secuenciación y toma de decisiones. Los juegos de azar resultan adecuados para fijar, afianzar y comprender concepto probabilísticos, además de inducir razonamiento deductivo.

Los juegos educativos, recreativos y colectivos son un recurso fundamental que interviene en el aprendizaje de los jóvenes con diversidad funcional; además de constituir un medio de enseñanza que puede ser aprovechado de manera efectiva en el proceso cognoscitivo, con mayor valor potencial si el juego es creado por la inspiración del estudiante, quien inventa el proceso, sus elementos y normativa de ejecución. En la enseñanza de la estadística y su rama de probabilidades, el uso de juegos tiene mayor justificación por cuanto los conceptos primigenios de probabilidad tuvieron su origen en la actividad lúdica de los siglos XVI y XVII, una época en que la base del cálculo de probabilidades surge a partir del lanzamiento de dados, en estudios sobre las posibilidades de ganar en apuestas de juegos de azar.

\section{DISEÑO METODOLÓGICO}

Se sometió a un proceso de experimentación a 45 estudiantes, quienes ejecutaron cuatro tareas organizadas en dos "prácticas". Las actividades se orientaron a la resolución de problemas, planteamiento de problemas, o ambos objetivos a la vez. Estas acciones estaban dirigidas a inducir en los alumnos, la aplicación de sus conocimientos probabilísticos; y a la generación de destrezas y estrategias de pensamiento adecuadas a las situaciones planteadas.

La enseñanza de los contenidos probabilísticos, a los que se refirieron las prácticas propuestas, se realizó previamente a éstas, 
durante cuatro sesiones de teoría de noventa minutos cada una; impartidas por el mismo profesor; y cinco de práctica de resolución de problemas, de cincuenta minutos cada una, ofrecidas por distintos profesores. Los contenidos estaban relacionados con la noción de experimento aleatorio, espacio muestral y sucesos; conceptos e interpretaciones de la probabilidad; definición axiomática y consecuencias de los axiomas; noción de espacio muestral finito, probabilidad condicionada; independencia y teorema de la multiplicidad, y finalmente, los teoremas de probabilidad total y de Bayes.

A los 45 estudiantes se les realizó un pre test evaluado. Posteriormente, se les motivó a participar en la invención de un juego didáctico, el cual, una vez ideado, se aplicó un pos test evaluativo valorativo. Finalmente se compararon resultados para determinar posibles bondades de la propuesta.

A los participantes se les prescribió algunas condiciones normativas a tener en consideración para la creación del juego. El mismo debía ser: un juego de azar de mesa didáctico; de tamaño $40 \mathrm{cms}$. x $30 \mathrm{cms}$. contener instrucciones y reglas claras, indicar objetivos del juego, número de participantes, mostrar un atractivo diseño de caratula y asignar un nombre que sugiera su orientación. Así mismo, la aleatoriedad debía estar basada en extracción de fichas, o cartas; lanzamiento de dados o monedas, entre otros procedimientos generados por la incertidumbre de su ejecución. Una vez caracterizados los eventos de azar, el alumno debió identificar y calcular la probabilidad de ocurrencia de los diferentes eventos aleatorios presentes durante la realización del juego, incluyendo diversos niveles de dificultad creciente.

Esta actividad inventiva exigió que el alumno formulase preguntas derivadas del desempeño funcional del juego; además de resolver y mostrar las respuestas correspondientes.

El ingenioso producto lúdico debió ser realizado por un grupo integrado por hasta 5 cursantes; y ser presentado en un plazo de entrega previamente estipulado. De esta manera, se estimuló la creación de juegos de naturaleza aleatoria, inéditos, para divertirse, pero fundamentalmente como instrumento para aprender probabilidades. Una actividad didáctica desafiante, por cuanto exigió a cada grupo de alumnos, la creación de un juego a partir de cero.

Como objetivos del juego se plantearon los siguientes:

- Reforzar los conocimientos teóricos de probabilidades impartidos en clase.

- $\quad$ Desarrollar destrezas para definir los espacios muestrales de diferentes ex perimentos aleatorios.

- Conceptualizar los sucesos mutua mente excluyentes e independientes.

- Inducir en los participantes un apren dizaje significativo de las teorías de probabilidades.

\section{RESULTADOS Y DISCUSIÓN}

\section{PRETEST}

En la aplicación del Pretest se realizó una evaluación cualitativa, donde se pudo describir las fallas encontradas en las evaluaciones, tales como, errores en la interpretación del Teorema de Bayes, errores en la definición del espacio muestral, errores de fórmulas, interpretaciones erradas de los sucesos independientes y mutuamente excluyentes, etc. También se asignó una calificación cuantitativa, como se indica a continuación: 
No. 1

Enero-Diciembre 2014 ISSN 1794-8231

\section{Fallas encontradas en el pretest}

\begin{tabular}{|l|c|}
\hline Uso incorrecto del teorema de Bayes & 43 \\
\hline $\begin{array}{l}\text { La intuición como predominio sobre el análisis matemático del } \\
\text { experimento }\end{array}$ & 40 \\
\hline Dificultad para imaginarse el experimento aleatorio planteado & 38 \\
\hline $\begin{array}{l}\text { Interpretación errada de los sucesos mutuamente excluyentes e } \\
\text { independientes }\end{array}$ & 34 \\
\hline Dificultad para definir el espacio muestral & 32 \\
\hline Uso indebido de las formulas de probabilidades & 27 \\
\hline $\begin{array}{l}\text { Una vez calculada la probabilidad, ausencia de la respectiva } \\
\text { interpretación }\end{array}$ & 21 \\
\hline Desconocimiento de las cartas de póker, domino, barajas españolas & 14 \\
\hline \multicolumn{1}{|c|}{ Fallas en las teorías de conjuntos y sus aplicaciones } & 14 \\
\hline \multicolumn{1}{|c|}{ Fallas en las operaciones básicas de fracciones } & 12 \\
\hline
\end{tabular}

\section{POSTEST}

Luego de la elaboración del juego y de su exposición, a los estudiantes se les realizó un pos test, y se observaron las mismas variables que en el pre test, encontrándose los siguientes resultados:

Se aplicó un test para probar la hipótesis de que la actividad lúdica mejora el rendimiento académico en los estudiantes. Se aplico una prueba de Bondad de ajuste a las notas obtenidas encontrándose que los datos no se distribuyen de acuerdo a la distribución normal. Se procedió a aplicar métodos no paramétricos escogiéndose la prueba de Wilcoxon para pares relacionados. Los resultados se muestran en anexo.

\begin{tabular}{|c|c|}
\hline Fallas encontradas en el postest & Postest \\
\hline Uso incorrecto del teorema de Bayes & 17 \\
\hline $\begin{array}{l}\text { La intuición como predominio sobre el análisis matemático del } \\
\text { experimento }\end{array}$ & 22 \\
\hline Dificultad para imaginarse el experimento aleatorio planteado & 9 \\
\hline $\begin{array}{l}\text { Interpretación errada de los sucesos mutuamente excluyentes e } \\
\text { independientes }\end{array}$ & 5 \\
\hline Dificultad para definir el espacio muestral & 12 \\
\hline Uso indebido de las formulas de probabilidades & 3 \\
\hline $\begin{array}{l}\text { Una vez calculada la probabilidad, ausencia de la respectiva } \\
\text { interpretación }\end{array}$ & 1 \\
\hline Desconocimiento de las cartas de póker, domino, barajas españolas & 0 \\
\hline Fallas en las teorías de conjuntos y sus aplicaciones & 6 \\
\hline Falta de motivación para la realización de la actividad & 1 \\
\hline Fallas en las operaciones básicas de fracciones & 0 \\
\hline
\end{tabular}




\section{CONCLUSIONES}

- Se logró participación activa de todos los estudiantes.

- La presentación de juegos inéditos, mostró el potencial creativo de los alumnos, para dar rienda suelta a su imaginación y a su capacidad para concretar ideas materializadas en invenciones tangibles.

- Los estudiantes se sintieron motivados para la realización del juego y aportaron instrumentos válidos para experimentar con sucesos aleatorios y pensar probabilísticamente.

- En la creación del juego, consolidaron los conceptos básicos de probabilidad, por cuanto ellos planteaban las preguntas y debían resolverlas.

- Se puso de manifiesto el ingenio, la inventiva y la creatividad propia de los estudiantes de Ingeniería Industrial y de la carrera de Mercadeo, para inventar desde inicio diversos juegos de azar y su pertinencia al objetivo de estudio.

- Desarrollaron habilidades para reconocer y definir los espacios muestrales.

- Desplegaron imaginación para plantear los experimentos aleatorios mostrados en los juegos.

- Lograron un aprendizaje significativo a través de una actividad divertida.

- El juego permitió orientar a los alumnos a expresar sus inquietudes e ideas sobre las temáticas referidas; además, facilitó a los participantes, el logro de aprendizajes sobre la teoría de probabilidades.

- El juego permitió una interacción exitosa entre el facilitador y los alumnos. Estos últimos mostraron una conducta adecuada, participaron de manera voluntaria, trabajaron organizadamente, exhibiendo respeto a las normas y decisiones.
- La estrategia educativa implementada permitió desarrollar en los participantes procesos cognitivos como la observación, comparación memorización, manejo de instrucciones e integración de la teoría y la práctica.

- Los alumnos lograron un mayor número de objetivos, según los resultados obtenidos en el pos test, después de aplicar el juego, y las actividades de cierre, observándose una reducción significativa de ideas erróneas y temores sobre la estimación de probabilidades.

\section{REFERENCIAS BIBLIOGRÁFICAS}

Ausbel D. (2009). Teoría del Aprendizaje significativo. Una perspectiva cognitiva. Ed. Trillas.México.

Martínez L. (2010). Lúdica como estrategia didáctica. Universidad Autónoma de Guadalajara. México.

Piaget J. (1986). La Formación del Símbolo en el niño. México: Fondo de Cultura Económica.
Enero-Diciembre 2014 ISSN 1794-8231

PP: 66-73 
Eco.Mat

No. 1
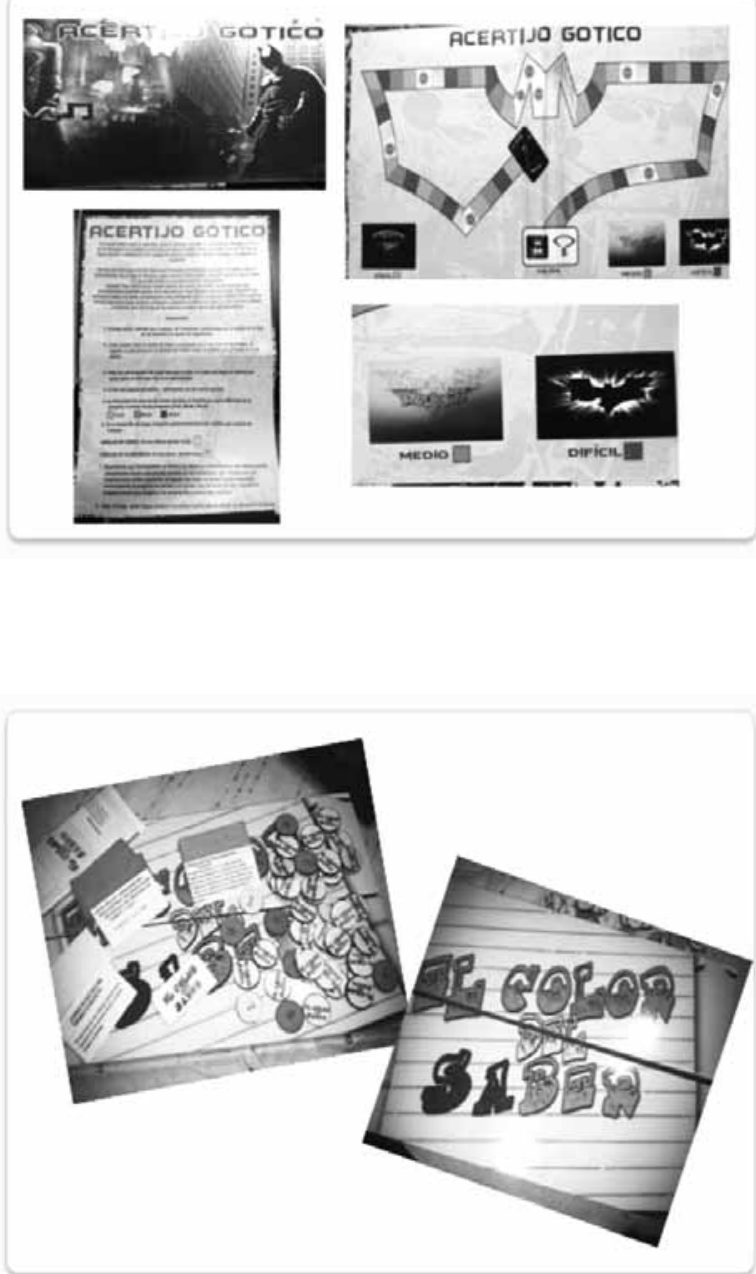

Reto al ingenio probabilistico: una propuesta didactica para la enseñanza-aprendizaje de las probabilidades

\section{ANEXOS \\ MUESTRA DE ALGUNOS DE LOS JUEGOS REALIZADOS}

\section{ACERTIJO GOTICO}

Es un juego que hace referencia a la serie de Batman, donde los jugadores deben resolver acertijos que conllevan diferentes cálculos de probabilidades, de situaciones donde los protagonistas son los personajes de la serie.

Existen distintos niveles de complejidad, los cuales deben ser superados con el avance del juego.

\section{EL COLOR DEL SABER}

Este juego consta de fichas de colores que tienen marcado un número y un color. Para cada color hay un grupo de tarjetas con preguntas, de acuerdo a cierto nivel de dificultad. El número representa los puntos que ganará el jugador si responde correctamente la pregunta. Los participantes escogen una ficha de las dispuestas en un tablero; deben voltearla y contestar la pregunta correspondiente. El juego lo ganará el jugador que acumule más puntos.

\section{BEISBOL EXTREMO}

Es un juego referido al Béisbol. Los alumnos realizaron las figuras y diseñaron el cartón simulando el campo del juego. La jerga del juego incluye los términos de un partido, como ponchado, carrera, strike. Un jugador anotará carrera para su equipo si es capaz de resolver los problemas referentes al cálculo de probabilidades que se le van preguntando en cada base señalada en el cartón. Se le cantará un strike por cada pregunta que falle en responder durante su turno al bate. Tres fallas ocasionan un "ponche". Para marcar carrera deberá contestar preguntas correspondientes a las tres bases dibujadas en el tablero. Cada base corresponde a un nivel de dificultad. 


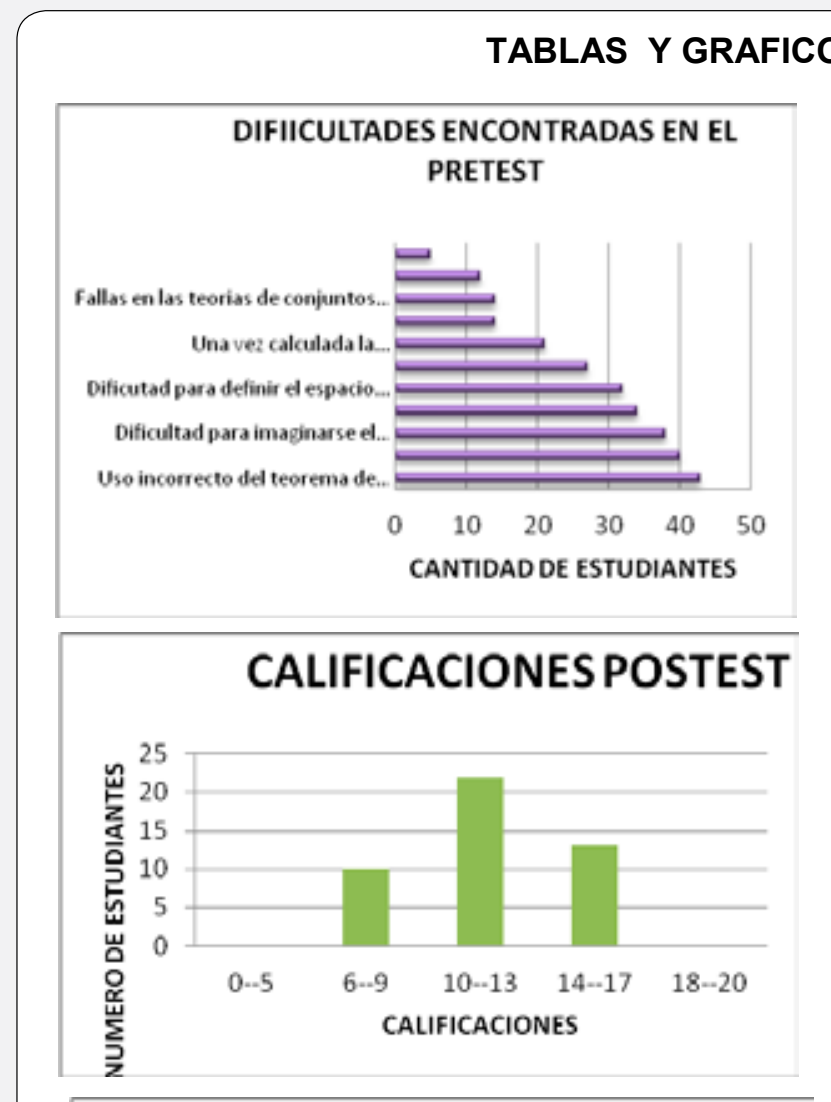

Enero-Diciembre 201
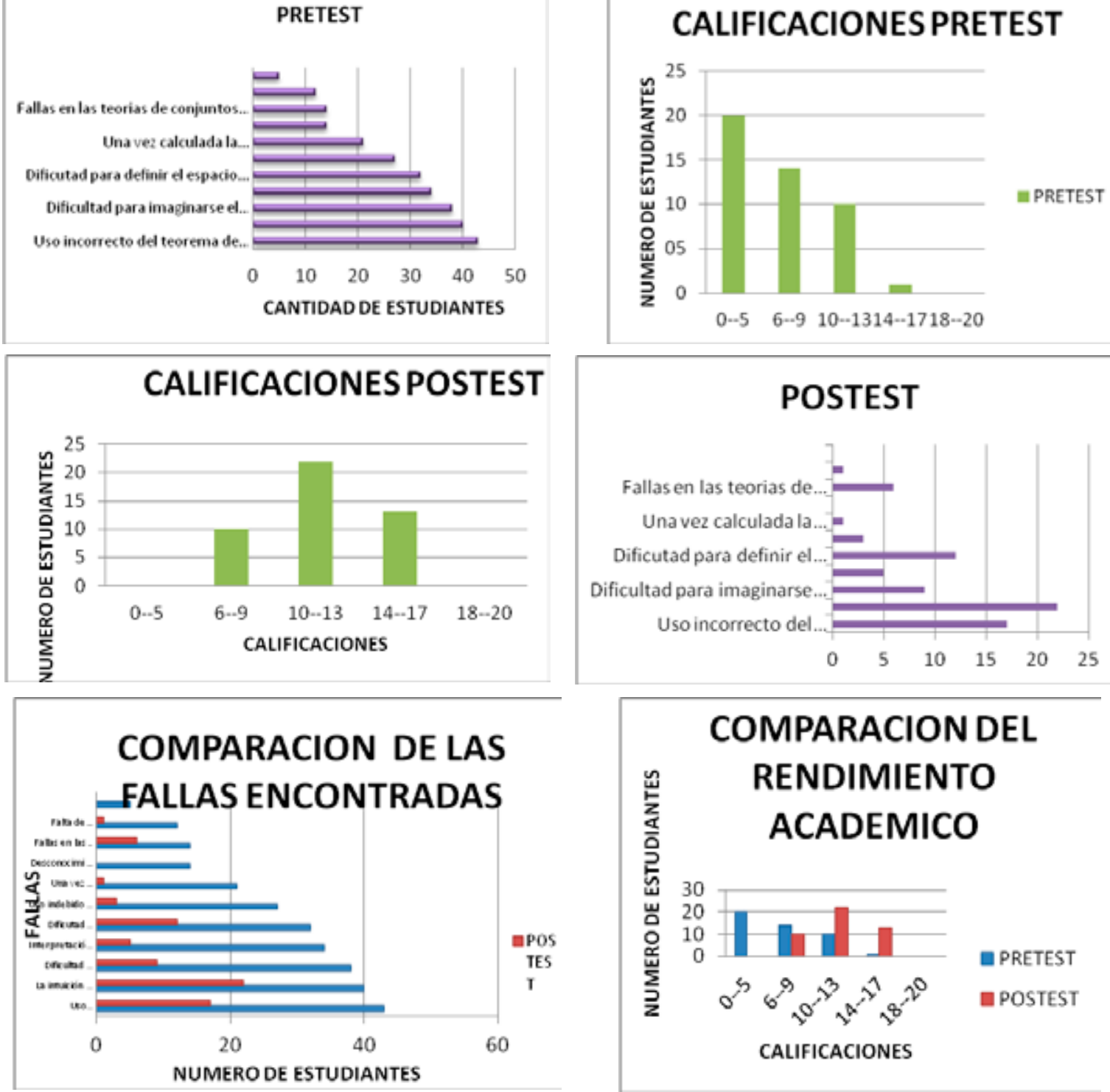

\begin{tabular}{|c|c|c|c|c|}
\hline \multicolumn{5}{|c|}{ PRUEBA DE WILCOXON PARA PARES RELACIONADOS } \\
\hline GRUPO & TAMAÑO & $\mathrm{W}$ & $\mathrm{U}$ & ESTADISTICO \\
\hline 1.PRETEST & 45 & 1291.5 & 256.5 & \multirow[b]{2}{*}{$\mathrm{U}=265.5$} \\
\hline 2.POSTEST & 45 & 2793.5 & 1758.5 & \\
\hline Valor tabla & & 4085 & & \\
\hline Significancia & 0.05 & & & $\mathrm{Wt}=276$ \\
\hline \multicolumn{5}{|c|}{$\begin{array}{c}\text { Como } U=265.5 \text { es menor a Wt=276 Se Rechaza Ho. Por lo tanto se } \\
\text { puede inferir que el juego mejora el aprendizaje. }\end{array}$} \\
\hline
\end{tabular}

